\title{
Efficacy of omalizumab in reducing latex allergy
}

\author{
Arianna Aruanno, Raffaella Chini, Eleonora Nucera \\ Allergy Unit, Fondazione Policlinico Universitario A. Gemelli IRCCS, Rome, Italy
}

Adv Dermatol Allergol 2021; XXXVIII (5): 921-923

DOI: https://doi.org/10.5114/ada.2021.110072

Omalizumab, a humanized monoclonal antibody that binds the $\mathrm{CH} 3$ domain near the binding site for the high affinity type-I lgE Fc receptors of human IgE, can neutralize free IgE and inhibit the lgE allergic pathway [1].

The current indications for treatment with omalizumab are limited to severe persistent allergic asthma and chronic spontaneous urticaria (CSU) when it is not controlled by conventional anti-H1 treatment at the maximum dosage.

In recent years, several studies [1] have shown that omalizumab is effective in a large number of IgE-mediated and non-IgE-mediated disorders, indicating that omalizumab treatment acts on many levels and not only on immunoglobulins E [2].

After diagnosis, prevention is the standard and best measure, but the strict avoidance is often impossible. In selected cases, the sublingual immunotherapy may be a therapeutic approach able to influence the long-term natural history of latex allergy $[3,4]$.

We showed the case of a young boy affected by severe persistent allergic asthma and rhinitis who presented with mild-moderate symptoms after latex exposure. The boy started omalizumab therapy to treat his severe asthma and, after 6 years of this treatment, we evaluated again the latex allergy. In the end, we observed negative challenges and an important decrease in all allergy parameters.

We describe the case of an 11-year-old boy referred to our Allergy Unit in 2011 reporting moderate rhinitis and severe persistent asthma (GINA step 4) and 4 episodes of palpebral and labial angioedema after contact with latex balloons during the last 3 years.

Based on the anamnesis, we performed a complete allergological work-out.

The skin prick tests (SPTs) were performed with the latex extract (Alk-àbello, Milan, Italy), major inhalant extract and histamine $10 \mu \mathrm{g} / \mathrm{ml}$ and saline solution (Lofarma, Milan, Italy) according to the EAACI recommendations [5]. The assays of serum total IgE, major inhalant allergens and latex specific IgE were then performed by UniCAP System (Pharmacia, Uppsala, Sweden) and the latex basophil activation test (BAT) was assessed by FACSCanto flow cytometer (BD Biosciences). The patient performed also a basal and after bronchodilator spirometry according to the standardized procedures [6]. In the end to confirm the latex allergy, the patient underwent cutaneous, nasal and conjunctival challenge tests [7].

So, the allergological evaluation showed positive SPTs and IgE value to dermatophagoides pter., grasses, olive tree, pellitory and cat epithelium. The basal spirometry demonstrated a severe flow obstruction reversible after bronchodilator.

Moreover, we found a $5.0 \mathrm{~mm}$ wheal for the latex extract, latex IgE of $5.10 \mathrm{U} / \mathrm{ml}$, recombinant latex allergens Hev b 6.01 and Hev b 6.02 both of 5.17 kUA/l. The latex challenges were all positive.

Because of his respiratory instability, the patient was initially treated by high-dose CSI/LABA, antileukotrienes and antihistamines. Because of persistent severe asthma exacerbations that needed a high dose of oral corticosteroids, in 2012, the young patient started omalizumab treatment (300 mg monthly) according to guidelines.

As regarding the latex allergy, since he and his family did not have difficulties avoiding latex, we decided to not begin a latex immunotherapy and recommended that they arrange medical visits and surgical interventions only in the "latex-free" environment.

Thanks to omalizumab therapy, he reached a good control of his rhinitis and bronchial asthma (as demonstrated by an increase in ACT score and enhancement of spirometry parameters) and he did not have any exacerbations requiring emergency therapies.

During this treatment, the patient did not report clinical symptoms after any accidental contact with latex. After 6 years the patient's allergological evaluation was repeated to monitor the possible latex immunological and allergological features changes (Table 1).

Address for correspondence: Arianna Aruanno MD, PhD, Allergy Unit, Fondazione Policlinico Universitario A. Gemelli IRCCS, Largo F. Vito, 1 - 00168 Rome, Italy, phone: +39 3282190241, fax: 06 30156999, e-mail: aarianna@hotmail.it, arianna.aruanno@policlinicogemelli.it Received: 24.11.2019, accepted: 31.12.2019. 
Table 1. Patient's allergological exam before and after omalizumab treatment

\begin{tabular}{lcc}
\hline Parameter & Before omalizumab treatment & After omalizumab treatment \\
\hline SPTs (wheal diameter) & $5 \mathrm{~mm}$ & $3 \mathrm{~mm}$ \\
\hline slgE: & & $2.03 \mathrm{U} / \mathrm{ml}$ \\
\hline Latex (k82) & $5.10 \mathrm{U} / \mathrm{ml}$ & 0.03 \\
\hline Hev b1 & 0.03 & 0.03 \\
\hline Hev b3 & 0.04 & 0.01 \\
\hline Hev b5 & 0.02 & Neg \\
\hline Hev b 6.01 & $5.17 \mathrm{kUA} / \mathrm{l}$ & $1.96 \mathrm{U} / \mathrm{ml}$ \\
\hline Hev b 6.02 & $5.17 \mathrm{kUA} / \mathrm{l}$ & 0.02 \\
\hline Hev b8 & 0.02 & 0.02 \\
\hline Hev b9 & 0.03 & 0.03 \\
\hline Hev b11 & 0.04 & Non responder \\
\hline BAT latex & Non responder & Negative \\
\hline Cutaneous challenge: & & Negative \\
\hline Vinyl glove-wearing & Positive & Negative \\
\hline Sterile glove-wearing & Positive & Negative \\
\hline Non sterile glove-wearing & Positive & Negative \\
\hline Nasal challenge with the latex extract & Positive & \\
\hline Conjunctival challenge with the latex extract & Positive & \\
\hline
\end{tabular}

The efficacy and good safety profile of omalizumab in patients with severe allergic asthma and CSU unresponsive to standard therapy are well known resulting in FDA approval for use in those diseases.

In recent years, several studies have shown that omalizumab is effective also in many other allergic diseases. These findings have improved our understanding that treatment acts on many levels and not only neutralizing free lgE and inhibiting the IgE allergic pathway. In fact, it is emerging other roles of omalizumab in regulating levels of inflammatory proteins and also in regulating levels of vitamin $D$, homocysteine, d-dimer, albumin and SApo-2L [2].

However, the role of omalizumab in IgE-mediated diseases remains indisputable.

In reality, different mechanisms of action of omalizumab were also hypothesized, which could explain its use off-label in many diseases: it reduces mast cell releasability, activity of IgG autoantibodies against FceRI and IgE, activity of intrinsically "abnormal" IgE, and activity of IgE autoantibodies against an autoantigen [8].

In our case, thanks to omalizumab, we saw a reduction in latex sensitization confirmed by a decrease in latex positivity on IgE tests. We hypothesize it could be due to omalizumab binding the Fc3 sub-unit of free IgE molecules: through this mechanism, the reduced binding of free specific IgE to the high-affinity IgE receptor (FceRI), leads to a reduced density of specific IgE on the surface of mast cells and basophils and to downregulation of the receptor itself.
A similar result comes from the literature in a placebocontrolled trial that included 18 latex-allergic health care workers randomly treated with either omalizumab or placebo. Data showed a statistically significant reduction in conjunctival and cutaneous responses to latex after omalizumab treatment [9].

In another recent case report [10], omalizumab also seems to improve latex-induced contact urticaria. In this case omalizumab might have induced the depletion of FceRI in skin mast cells leading to a favourable response in latex contact urticaria.

These studies corroborate our hypothesis of a possible role of omalizumab in the treatment of latex allergy and open to a future new indication of omalizumab in therapy.

In conclusion, the findings of this report show that omalizumab has a relevant role in reducing symptoms and IgE sensitization in a patient affected by latex allergy. This hypothesis is corroborated by previous scientific research projects in literature $[9,10]$.

Further studies on a larger scale are certainly needed to validate this new possible use of omalizumab and to evaluate the persistence of latex tolerability after discontinuation of omalizumab.

\section{Acknowledgments}

The authors are responsible for the content and the writing of this paper. Arianna Aruanno and Raffaella Chini contributed equally to the work. 


\section{Conflict of interest}

The authors declare no conflict of interest.

\section{References}

1. Incorvaia C, Mauro M, Makri E, et al. Two decades with omalizumab: what we still have to learn. Biologics 2018; 12: 135-42.

2. Yalcin AD. An overview of the effects of anti-lgE therapies. Med Sci Monit 2014; 20: 1691-9.

3. Turjanmaa K. Incidence of immediate allergy to latex gloves in hospital personnel. Contact Dermatitis 1987; 17: 270-5.

4. Sridharan K, Sivaramakrishnan G. Sublingual immunotherapy in patients with latex allergy: systematic review and meta-analysis of randomized controlled trials. J Dermatolog Treat 2017; 28: 600-5.

5. Dreborg S. Allergen standardization and skin test. EAACI position paper. Allergy 1993; 48 (Suppl. 14): 49-82.

6. Miller MR, Hankinson J, Brusasco V, et al.; ATS/ERS Task Force. Standardisation of spirometry. Eur Respir J 2005; 26: 319-38.

7. Nucera E, Pollastrini E, Sabato V, et al. Challenge tests in the diagnosis of latex allergy. Int I Immunopathol Pharmacol 2010; 23: 543-52.

8. El-Qutob D. Off-label uses of omalizumab. Clin Rev Allergy Immunol 2016; 50: 84-96.

9. Leynadier F, Doudou O, Gaouar H, et al. Effect of omalizum$a b$ in healthcare workers with occupational latex allergy. J Allergy Clin Immunol 2004; 113: 360-61.

10. Di Leo E, Calogiuri G, Macchia L, Nettis E. Use of omalizumab in uncontrolled chronic spontaneous urticaria also improved latex-induced contact urticaria. J Allergy Clin Immunol Pract 2019; 7: 300-2. 